\title{
Measurement of dynamic deformations using a path-unbalance Michelson-interferometer- based optical fiber sensing device
}

\author{
Sandra LLoret \\ Pramod Rastogi, FELLOW SPIE \\ Swiss Federal Institute of Technology \\ Lausanne \\ Applied Computing and Mechanics \\ Laboratory (IMAC) \\ $\mathrm{CH}-1015$ Lausanne, Switzerland \\ E-mail: pramod.rastogi@epfl.ch \\ Luc Thévenaz \\ Swiss Federal Institute of Technology \\ Lausanne \\ Metrology and Photonics Laboratory \\ $\mathrm{CH}-1015$ Lausanne, Switzerland \\ Daniele Inaudi, MEMBER SPIE \\ SMARTEC SA \\ Via Pobbiette 11 \\ CH-6928 Manno, Switzerland
}

\begin{abstract}
A novel demodulation technique for performing dynamic deformation measurements using a path-unbalanced Michelson interferometer is reported. The method is based on the rf amplitude modulation of a low-coherence source, and demodulation is achieved by tracking in the frequency domain the position of the minimum of the detected intensity. This technique is particularly suitable for deformation measurements in civil engineering structures where deformations of the order of few millimeters over the sensor length are expected. The method features a measurement range of at least $10 \mathrm{~mm}$, sensitivity better than $10 \mu \mathrm{m}$, and dynamic deformation measurements with a bandwidth up to $100 \mathrm{~Hz}$. (c) 2003 Society of Photo-Optical Instrumentation Engineers. [DOI: 10.1117/1.1543563]
\end{abstract}

Subject terms: optical fiber sensors; Michelson interferometer; deformation measurements; dynamic measurements; amplitude modulation; tracking system.

Paper 020241 received Jun. 12, 2002; revised manuscript received Aug. 14, 2002; accepted for publication Aug. 30, 2002.

\section{Introduction}

Rapid growth in demand for value-added techniques for health monitoring of structures has focused worldwide interest on optical fiber sensors as the provider of an effective solution to measurement problems. ${ }^{1}$ Recent advances in measurement technology have demonstrated that fiber optic sensors are suitable for the monitoring of full-scale civil structures. ${ }^{2}$ When installed on structures, these sensors allow for monitoring important parameters such as deformations, temperatures, $\mathrm{pH}$ values, and gas concentrations. Given the exceptional stability and the flexibility with which large structures can be instrumented with an optical fiber sensing network, ${ }^{3,4}$ it can be reasonably expected that optical fiber sensors will become the backbone of measurement systems dedicated to monitoring the state of the infrastructure worldwide. Notwithstanding this enormous potential, present developments are far from fulfilling the promise that the fiber optics sensor technology holds in structural health monitoring.

In this context, the recent development in our facility of a long-gauge-length fiber optic sensor system, better known by the acronym SOFO, can be considered as a significant achievement in the area of structural strain monitoring in civil engineering structures. The system has the potential of monitoring structures over long periods of time, and has been successfully embedded or surface-mounted in a variety of materials such as concrete, steel, and mortar. ${ }^{5,6}$ The sensor, based on low-coherence interferometry, uses a Michelson interferometer with an initial path unbalance of about $1 \mathrm{~mm}$ between its arms. Of the two arms, one, the measurement arm, in mechanical contact with the structure, is used to follow the structure's deformation, and the second, the reference arm, is free and serves for compensating temperature variations. The analyzing unit of the sensor integrates a second scanning interferometer that is capable of introducing a known path-length difference between its two arms. From the point of view of performance, the sensor system has a resolution of $2 \mu \mathrm{m}$ and a measurement range of $5 \mathrm{~mm}$, and more than 2500 transducer units installed and operational for several years have demonstrated long-term stability.

Although the existing SOFO system, which is now also commercialized, is well suited for long-term assessment, the fact that each measurement takes about $10 \mathrm{~s}$ renders the system inadequate for monitoring the dynamic behavior of structures. Such an investigation would require the measurement and storing of many readings per second. Hence the present work was undertaken with the main objective of providing to the SOFO system the additional possibility of measuring the dynamic deformation of structures.

A variety of interferometric demodulation techniques have been successfully implemented for measuring small deformations at low frequencies. ${ }^{7-10}$ However, given the requirements of long measurement base and high performance from the dynamic analyzing unit, these techniques do not lend themselves well to adaptation to structural applications.

In beam modulation telemetry, the amplitude of light modulated at high frequencies (a few hundred megahertz to a few gigahertz) is transmitted from a laser source to the reflector. The reflected beam is collected by the receiving system, which monitors the light falling on it. The optical distance between the light source and reflector is determined through comparison of the phases of the modulated waveforms of the transmitted and reflected beams. ${ }^{11,12} \mathrm{Al}-$ though this approach is used for measuring large distances, a similar type of approach may be applied to a Michelson interferometer operated with a low-coherence source for 


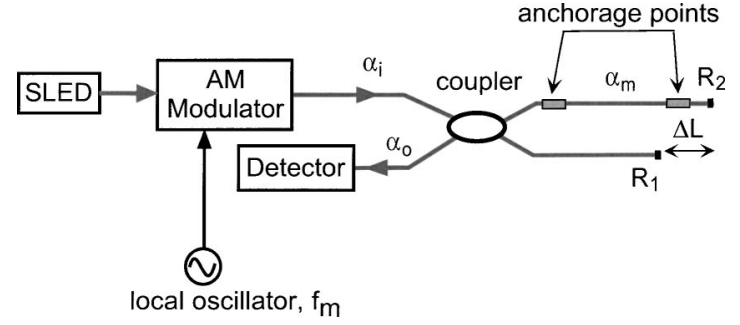

Fig. 1 Michelson configuration interrogated by amplitude modulated broadband source.

obtaining dynamic measurements. The aim of this paper is to describe, implement, and demonstrate a novel concept for performing dynamic measurements on structures. The interferometric configuration features long-term static and dynamic deformation monitoring of civil engineering structures with a unique sensor network.

\section{Principle of Operation}

Let us suppose the light from an intensity-modulated lowcoherence source to be incident on a Michelson interferometer. Ideally, the interferometer has $100 \%$ reflection at the end mirrors, a $50 \%$ splitting ratio at the coupler, and no additional intensity losses in any of the optical fibers constituting the arrangement. A schematic diagram of the proposed setup is shown in Fig. 1. A Mach-Zehnder-based amplitude modulator is used to modulate the light emitted by a superluminescent light emitting diode (SLED), the light source employed in the setup. Assuming the voltage applied to the modulator to be sinusoidally modulated, the intensity injected into the interferometer is given by

$I_{i}(t)=I_{0}\left\{1+\cos \left[\frac{\pi}{V_{\pi}} a_{0} \cos \left(2 \pi f_{m} t\right)+\varphi_{B}\right]\right\}$,

where $I_{0}$ is the mean intensity, $f_{m}$ is the modulation frequency, $V_{\pi}$ is the voltage required to cause a phase retardation of $\pi$ between the two arms of the Mach-Zender modulator, $\varphi_{B}$ is the phase bias needed to set the device at the operating point, and $a_{0}$ is the amplitude of the applied modulation voltage.

The coupler recombines the beams reflected by the interferometer arms. The relative delay at the output is directly proportional to the path difference between the two beams in the interferometer. Since the optical path difference in the two arms is of the order of $1 \mathrm{~mm}$, which is larger than the coherence length of the source, only the superposition in intensity of the back-reflected signals is recovered at a detector placed at the output of the interferometer. The fact that we are not observing any interference is an advantage in that it saves us from taking into account the states of polarization of the interference beams. The detected intensity at the output of the sensor is given by

$$
\begin{aligned}
I_{D}(t)= & \frac{1}{2} I_{0}+\frac{1}{4} I_{0}\left(\cos \left[\frac{\pi}{V_{\pi}} a_{0} \cos \left(2 \pi f_{m} t\right)+\varphi_{B}\right]\right. \\
& \left.+\cos \left\{\frac{\pi}{V_{\pi}} a_{0} \cos \left[2 \pi f_{m}(t-\tau)\right]+\varphi_{B}\right\}\right) \\
= & A_{0}+A(t),
\end{aligned}
$$

where $\tau=2 n \Delta L / c$ is the flight-time difference between the two arms, $A_{0}$ is the dc component, $A(t)$ is the amplitude modulation of the detected intensity, $n$ is the refractive index of the fiber, and $c$ is the velocity of light in vacuum. If the relative delay between the two beams is an odd multiple of the modulation half period, the reflected beams will be out of phase and no modulation will appear at the output. On the other hand, if the relative delay is an even multiple of the modulation signal half period, the reflected beams are in phase and the modulated component reaches a maximum. This implies that by changing the relative delay between the two arms of the sensor, a succession of maxima and minima can be obtained in the modulated component, which contributes to transforming the phase lag between the two reflected rf signals into an amplitude change.

The effect of modulation can be quantified by calculating the root-mean-square value, rms, of the normalized ac component:

$$
\begin{aligned}
\mathrm{rms} & =\left[\left\langle\left(\frac{A(t)}{A_{0}}\right)^{2}\right\rangle-\left\langle\frac{A(t)}{A_{0}}\right\rangle^{2}\right]^{1 / 2} \\
& =\left\{\frac{1}{T} \int_{0}^{T}\left[\frac{A(t)}{A_{0}}\right]^{2} \mathrm{~d} t-\left[\frac{1}{T} \int_{0}^{T} \frac{A(t)}{A_{0}} \mathrm{~d} t\right]^{2}\right\}^{1 / 2},
\end{aligned}
$$

where \langle\rangle indicates the mean value. The normalized rms value at the quadrature working point of the modulator is expressed by

$$
\begin{aligned}
\mathrm{rms}= & \left\{\frac{1}{4}\left[1-J_{0}(\pi)\right]+\frac{1}{4}\left[J_{0}\left(\pi \sin \left(2 \pi f_{m} \tau / 2\right)\right)\right.\right. \\
& \left.\left.-J_{0}\left(\pi \cos \left(2 \pi f_{m} \tau / 2\right)\right)\right]\right\}^{1 / 2},
\end{aligned}
$$

where $J_{0}$ is the Bessel function of the zero order. The product of the modulation frequency and the flight-time difference between the fibers allows determining one of the parameters in which we are particularly interested. We define this parameter as $z$ :

$z=f_{m} \tau=\frac{2 n \Delta L f_{m}}{c}$

Figure 2 shows a plot of the rms value as a function of the modulation frequency for two different values of $\Delta L$. The minimum value of the curve is seen to shift towards higher frequencies with a decrease in $\Delta L$. Since the position of the minimum depends only on the path difference in the sensor and on the modulation frequency, the frequency shifts of the minimum arising from deformations can be related to corresponding changes in the path difference in the sensor. 


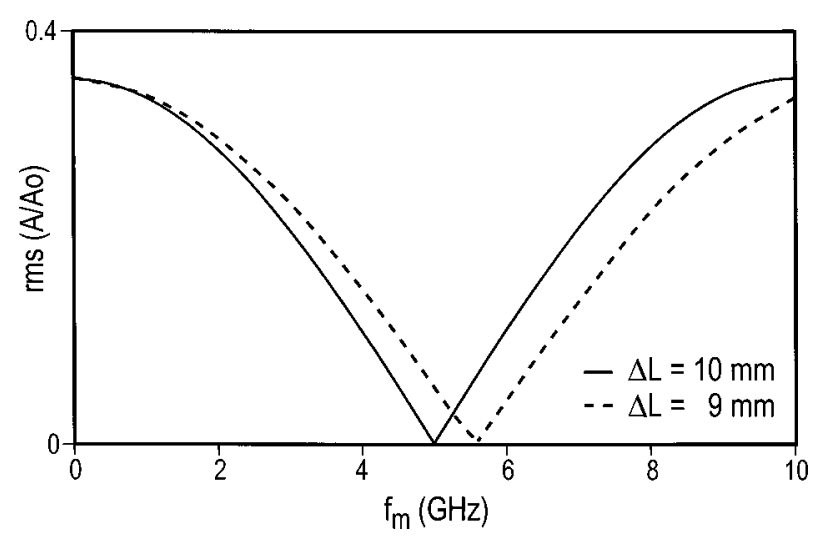

Fig. 2 Evolution of the modulated amplitude as a function of the modulation frequency for different $\Delta L$.

\section{System Considerations}

A compact sensor for dynamic deformation measurements is configured through integration of the principle outlined above in a SOFO sensor. In this section we focus our attention on considerations that seem to play an important role in determining the performance of the so configured sensor system. In Fig. $1, \alpha_{i}, \alpha_{o}$ denote the intensity losses in the fibers carrying light from the light source to the sensor system, and from the sensor system to the reading unit, respectively. Since the distance between the reading unit and the sensor can be of the order of few hundred meters in field applications, power losses due to bends or squeezes in the fibers may be important. Experimental tests have confirmed that changes in $\alpha_{i}$ and $\alpha_{o}$ are slowly varying functions of time.

The other performance factors are the reflectance values $R_{1}$ and $R_{2}$ of the mirrors in the reference and measurement arms, respectively. Although these mirrors are fabricated by depositing silver on the cleaved end facets of the fibers, and the obtained reflectance values are high enough, their values cannot be considered a priori as either equal $\left(R_{1}\right.$ $\left.\neq R_{2}\right)$ or ideal $\left(R_{1}, R_{2} \neq 1\right)$. The definitions of $R_{1}$ and $R_{2}$ are also assumed to take into account coupler imperfections that can slightly affect the splitting ratio.

Due consideration must also be given to macrobend losses in the measurement arm. Uncontrolled tight macrobends are formed in regions where the measurement fiber is glued to the anchor pieces (Fig. 3). Bending modifies the light-guiding property of the fiber, which results in a decrease in the light intensity flowing in the measurement arm of the sensor. Since the radius of fiber bending depends on the fiber deformation, the macrobending induces variable

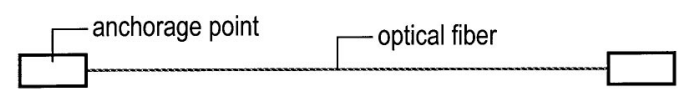

a)

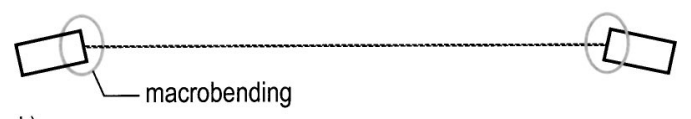

b)

Fig. 3 (a) A perfectly glued fiber. (b) In a real sensor, the fiber is not attached perfectly in line with the anchorage pieces.

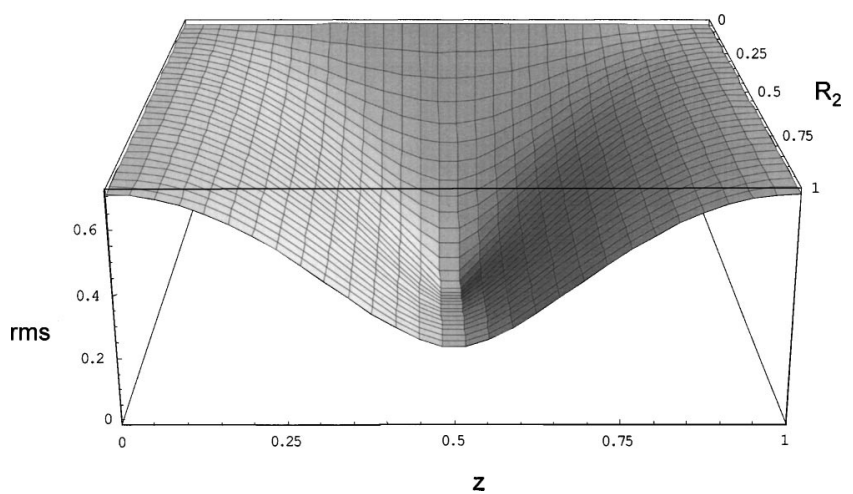

Fig. 4 Modulated amplitude as a function of parameters $z$ and $R_{2}$.

intensity losses in the light beam flowing in the measurement arm. This phenomenon is characterized by the quantity $\alpha_{m}$, which refers to intensity losses due to macrobends in a single trip of light through the measurement arm of the sensor. Herein we refer to these intensity losses as straininduced losses.

Taking into consideration the effects of these three factors on the performance of the sensor, the intensity detected at the output can be expressed as

$$
\begin{aligned}
I_{D}(t)= & \frac{1}{4} I_{0} \alpha_{i} \alpha_{o}\left(R_{1}+R_{2} \alpha_{m}^{2}\right) \\
& +\frac{1}{4} I_{0} \alpha_{i} \alpha_{o}\left(R_{1} \cos \left[\frac{\pi}{V_{\pi}} a_{o} \cos \left(2 \pi f_{m} t\right)+\varphi_{B}\right]\right. \\
& \left.+R_{2} \alpha_{m}^{2} \cos \left(\frac{\pi}{V_{\pi}} a_{o} \cos \left[2 \pi f_{m}(t-\tau)\right]+\varphi_{B}\right\}\right) \\
= & A_{0}+A(t),
\end{aligned}
$$

where the factor $\alpha_{m}^{2}$ takes account of round-trip straininduced losses.

\subsection{Effect of Reflectance Values $R_{1}$ and $R_{2}$}

Mirror reflectance values are fixed during the sensor fabrication and could safely be expected also to remain constant during measurements. In order to study their effect on the system, we suppose that

$R_{1}=R \quad$ and $\quad R_{2}=k R$.

where $0.75<k<1.3$ for a typically encountered value of reflectance. In this case, the rms value can be written as

$$
\begin{aligned}
\mathrm{rms}= & \left\{\frac{1+k^{2} \alpha_{m}^{4}}{\left(1+k \alpha_{m}^{2}\right)^{2}}\left[\frac{1}{2}-\frac{1}{2} J_{0}(\pi)\right]+\frac{k \alpha_{m}^{2}}{\left(1+k \alpha_{m}^{2}\right)^{2}}\right. \\
& \left.\times\left[J_{0}\left(\pi \sin \left(2 \pi f_{m} \tau / 2\right)\right)-J_{0}\left(\pi \cos \left(2 \pi f_{m} \tau / 2\right)\right)\right]\right\}^{1 / 2} .
\end{aligned}
$$

Figure 4 plots the variation of the rms value over a period as a function of $z$, for different values of $R_{2}$. The value $R_{1}=0.5$ is assumed to remain fixed. One notes from these 


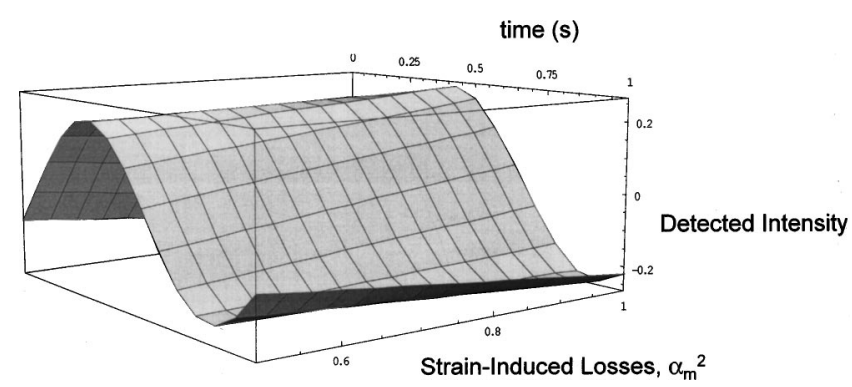

Fig. 5 Plot of detected intensity as a function of time and $\alpha_{m}^{2}$ for a real sensor.

curves that the minimum tends to become flatter with an increase in the difference between the reflectance values.

\subsection{Effect of Strain-Induced Losses, $\alpha_{m}^{2}$}

Radiation losses due to bends in optical fibers have received significant attention because of their influence on the power transmission capacity of optical fiber communication systems. The derivation of mathematical expressions for describing the behavior of bend losses is not straightforward, and the solution to this problem can be approached in different manners. ${ }^{13,14}$ Based on experimental observations, we have studied the parameter $\alpha_{m}^{2}$ in a qualitative manner and with a view to understanding its overall influence on the system.

Figure 5 shows the plot of the modulated component $A(t)$ as a function of time and $\alpha_{m}^{2}$, where we have assumed a fixed delay of $\tau=0.33 / f_{m}$ between the reflected intensities. The plot puts in evidence that changes in $\alpha_{m}^{2}$ have the same effect as that of introducing an unknown and variable phase shift in the detected wave. This also explains why $\Delta L$ cannot be recovered reliably by a phase detection technique.

One of the influences of the strain-induced losses is to cause the minimum to become flatter, as does nonequality of reflectance values. As an example, Figure 6 shows the response of the spectrum of a 2-m-long sensor with an initial path unbalance of $9 \mathrm{~mm}$, when the measurement arm is stretched by $5 \mathrm{~mm}$. The curve shifts towards lower frequencies with an increase in the path imbalance, and the effect of macrobend losses becomes apparent by way of the de-

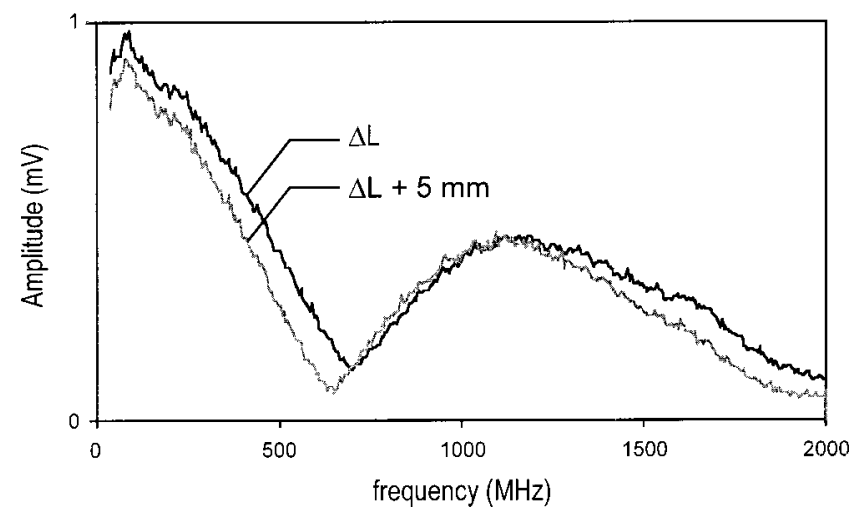

Fig. 6 Response of sensor spectrum to an elongation of $5 \mathrm{~mm}$. crease in the amplitude of the minimum. The losses introduced by stretching of the fiber tend to equalize the intensities reflected in each arm, and lead to a sharper minimum.

Since strain-induced losses cannot be easily controlled, their behavior will differ from one sensor to another. Tests performed with several sensors have shown that these losses can attain values as high as $0.25 \mathrm{~dB}$ through stretching the fiber in the measurement arm by amounts on the order of $1 \mathrm{~mm}$ - a value that is representative of the measurement range of the method-which is nonnegligible for an intensity-based method. The effect of a macrobend depends on the conditions under which the bend originates and the way the imposed deformation influences its evolution.

\section{Tracking of the Minimum}

We have noted in Sec. 2 that for a given path imbalance in the sensor, the minimum of the detected signal will be obtained for one precise value of the frequency modulation. The inverse is also true, that is, for a given value of frequency modulation, the minimum of detected intensity will be obtained for one precise value of the initial path unbalance in the sensor. This implies that one interesting possibility for measuring deformation variations in the sensor would be to monitor in the frequency domain the shift of the position of the intensity minimum with applied deformation. At the position of the minimum of the detected intensity, the relationship between the flight-time difference and the modulation frequency is given by

$f_{\min } \tau=f_{\min } 0.8 \frac{2 n}{c} \Delta L=0.5$.

The factor 0.8 takes into account the refractive index variation of the core when the fiber is strained. ${ }^{15}$ This factor has been confirmed through a number of calibration experiments. The path imbalance in the sensor can be calculated from

$\Delta L=\frac{6.4212 \times 10^{7}}{f_{\min }}$.

Expression (9) depends only on the characteristic parameters of optical fibers, which fortunately remain constant during measurements.

The inherent advantage of using this approach is that the position of the minimum is independent of strain-induced losses. However, the developments carried out in Sec. 3 do show that since the minimum tends to become flatter with increase in the value of $k \alpha_{m}^{2}$, the detection of its precise position would be prone to ambiguity.

\subsection{Principle of Operation}

The principle of operation for tracking the minimum of the detected signal is based on adding a frequency modulation to the carrier frequency $f_{m}$. Thus, the sensor input intensity produced by the superposition of amplitude and a frequency modulation can be written as 


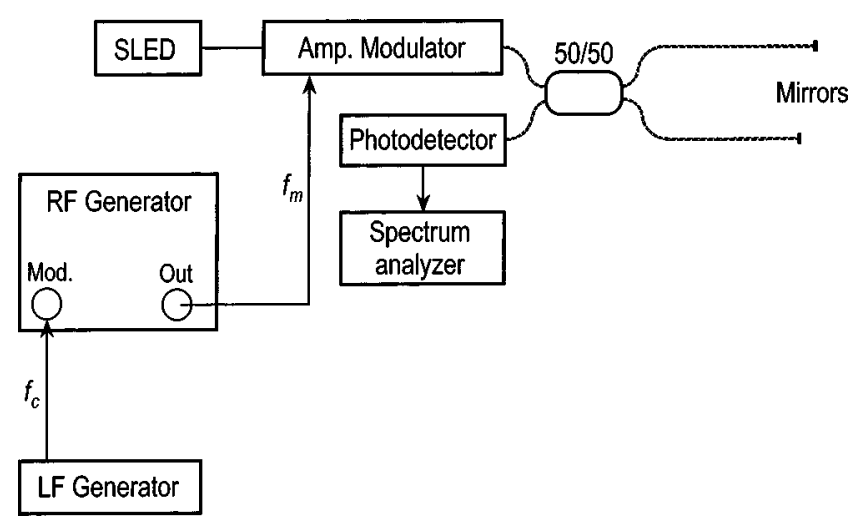

Fig. 7 Experimental arrangement for the minimum-position determination.

$$
\begin{aligned}
I_{i}(t)= & I_{0}\left(1+\cos \left\{\frac{\pi}{V_{\pi}} a_{0} \cos \left[2 \pi f_{m} t+\beta \sin \left(2 \pi f_{c} t\right)\right]\right.\right. \\
& \left.\left.+\varphi_{B}\right\}\right),
\end{aligned}
$$

where $\beta$, the modulation index of the frequency modulation, is given by

$\beta=\frac{\Delta f}{f_{c}}$,

$\Delta f$ is the maximum frequency shift, and $f_{c}$ is the frequency of the frequency-modulating signal. The basic configuration of the proposed setup is illustrated in Fig. 7. The $\mathrm{rf}$ carrier is generated with a Hewlett-Packard rf sweep generator, whose characteristic parameters are the central frequency $\mathrm{CF}$ and the frequency excursion $\Delta F$. A lowfrequency generator allows for introducing into the setup an external low frequency modulation. The modulated intensity at the sensor output is given by

$$
\begin{aligned}
I_{D}(t)= & \frac{1}{4} I_{0} R_{1} \cos \left\{\frac{\pi}{V_{x}} a_{0} \cos \left[2 \pi f_{m} t+\beta \sin \left(2 \pi f_{c} t\right)\right]\right. \\
& \left.+\varphi_{B}\right\}+\frac{1}{4} I_{0} R_{2} \alpha_{m}^{2} \cos \left(\frac { \pi } { V _ { \pi } } a _ { 0 } \operatorname { c o s } \left\{2 \pi f_{m}(t-\tau)\right.\right. \\
& \left.\left.+\beta \sin \left[2 \pi f_{c}(t-\tau)\right]\right\}+\varphi_{B}\right) .
\end{aligned}
$$

Figure 8 (a) shows the spectrum-analyzer trace of the sensor output obtained for the correct adjustment of the rf modulation frequency $f_{m}$ corresponding to the position of the minimum. Since the sensor used by us had a path unbalance of about $9.5 \mathrm{~mm}$, the position of the minimum would be at around $680 \mathrm{MHz}$. The frequency-modulated signal shows a frequency shift of $26 \mathrm{MHz}$ and a frequency of $10 \mathrm{kHz}$. The frequency scan at $f_{c}$ allows for an unambiguous identification of the form of the minimum. Figure 8(b) shows a spectrum-analyzer trace of the same sensor with the adjustment of rf modulation still far from its optimum position.
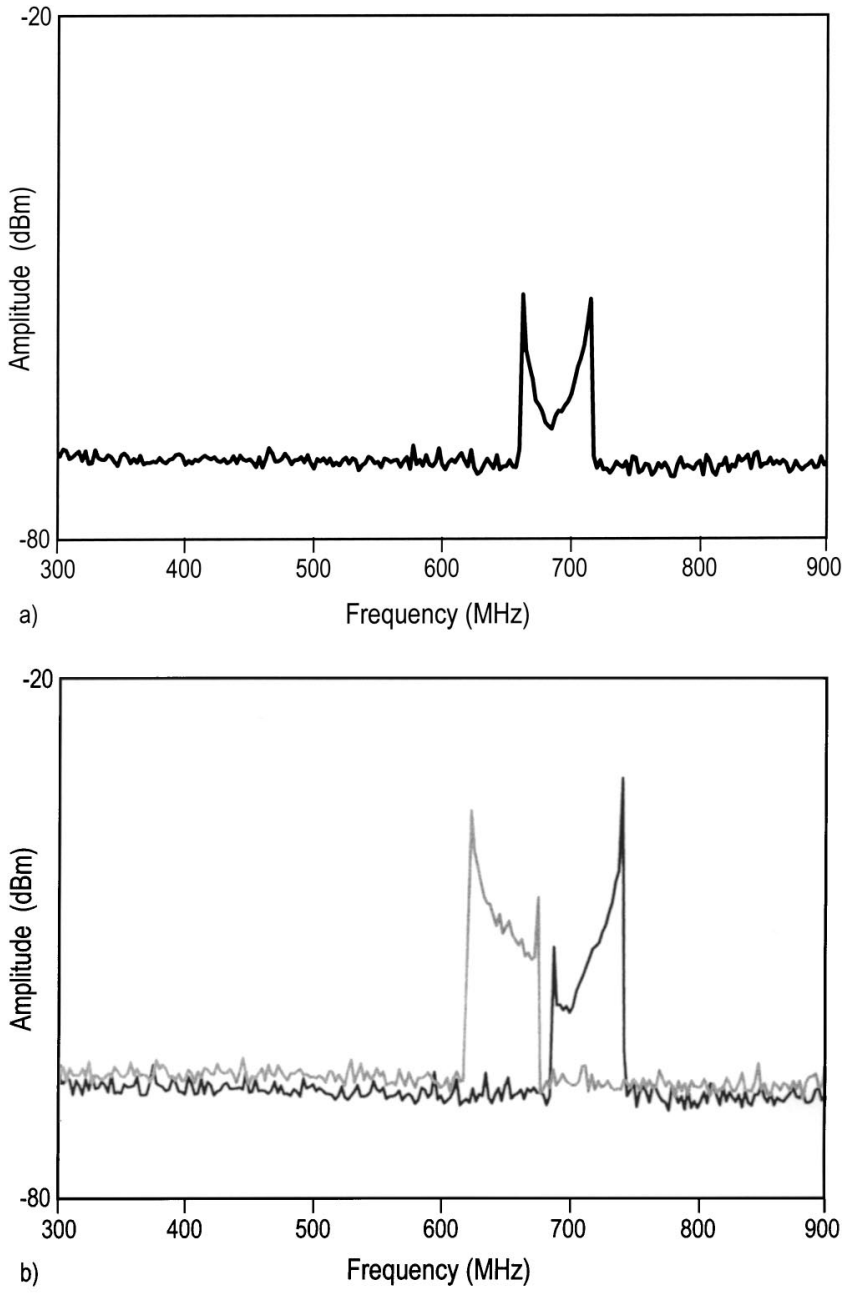

Fig. 8 Intensity spectrum of (a) the photodetector output at the minimum and (b) the photodetector output for carrier frequencies at both sides of the minimum position.

Figure 9 illustrates the behavior of the system in the frequency domain for different values of the carrier frequency $f_{m}$. When modulating on both sides of the minimum position, the frequency modulation generates a sinusoid at $f_{c}$ with amplitude proportional to the rate of change of the amplitude of the sensor response. At the minimum

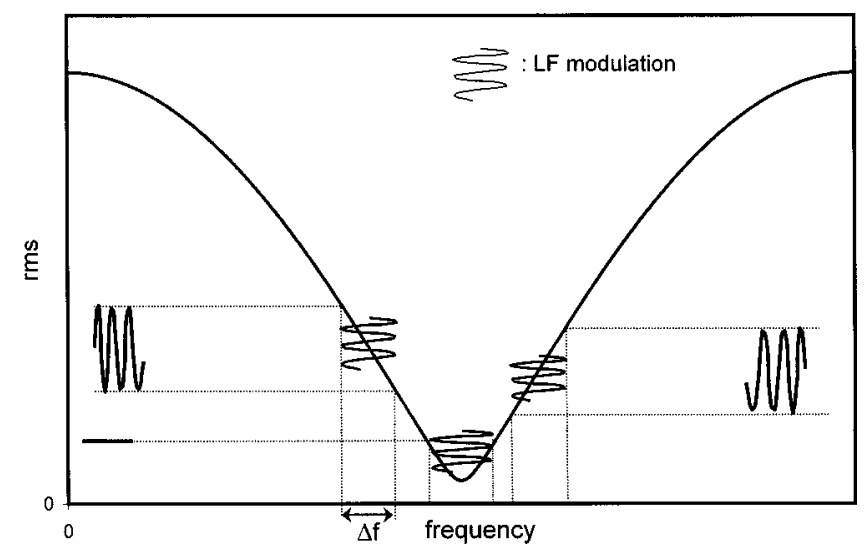

Fig. 9 Scheme of the operating principle. 


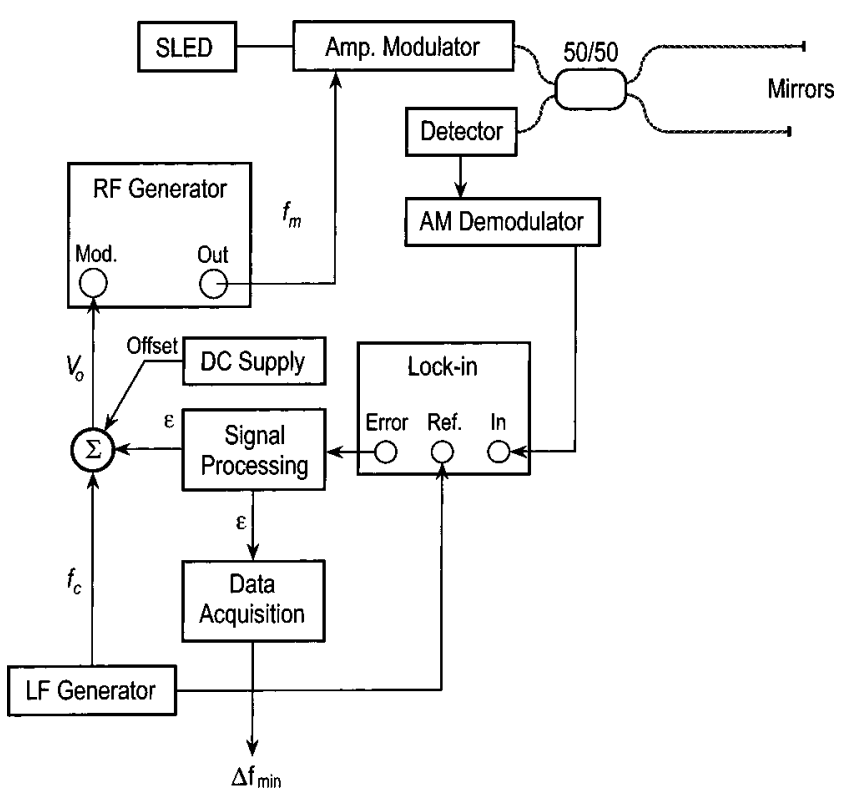

Fig. 10 Experimental setup of the tracking system.

position, this sinusoid has amplitude equal to 0 . Hence, to run the system smoothly, it is advisable to first generate a signal voltage proportional to the amplitude of the lowfrequency signal-in order to have an approximate appreciation of the position of the minimum-and then use this information for adjusting the position of the minimum.

\subsection{Demodulation Principle}

The demodulation is done in several steps. First, an envelope detector is used to eliminate the carrier frequency (amplitude demodulation). An advantage of the arrangement shown in Fig. 10 is that apart from amplitude demodulation, most of the processing it involves is done at low frequencies, which significantly contributes in simplifying the electronics needed to build such a system. A lock-in amplifier is next used to retrieve the amplitude of the lowfrequency signal (frequency demodulation). The lock-in generates a so-called error signal, which is proportional to the distance separating it from the position of the minimum. The purpose of doing this is to lock the system at the minimum frequency before calculating the frequency shifts from the error signal. Hence, a feedback loop is first inserted into the setup to monitor the position of the minimum frequency, which is followed by putting in place a system for automatic compensation of these changes. The feedback loop adds together the error signal obtained after integration and amplification from the lock-in, the lowfrequency modulation, and a certain offset. The resultant feedback voltage is

$V_{0}=G \times\left(A \sin 2 \pi f_{c} t+\right.$ offset $\left.+\varepsilon\right)$,

where $G$ is the total gain of the adder circuit, $A$ is the amplitude of the frequency-modulating signal, and $\varepsilon$ is the error signal from the lock-in obtained after integration and amplification.

Figure 11 shows the results obtained in steps of $50 \mu \mathrm{m}$ from a static scan performed with a 1.8-m-long sensor and

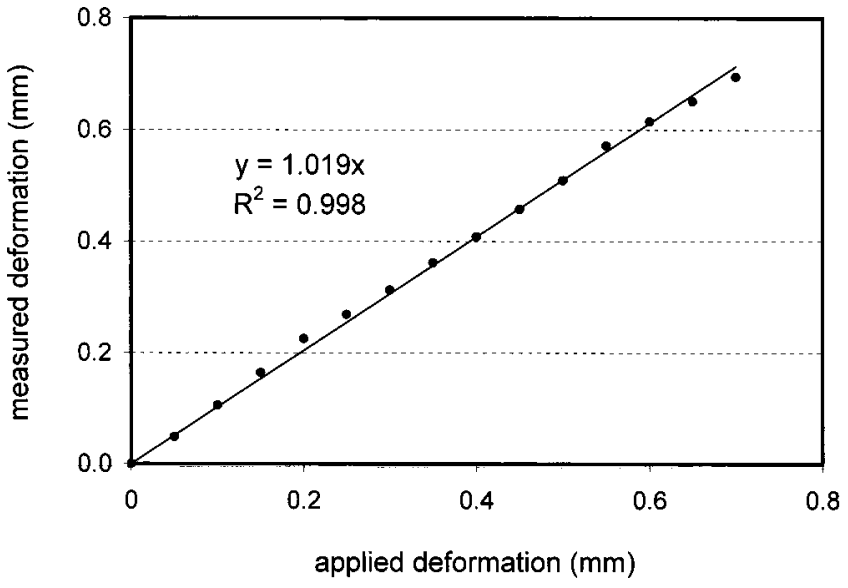

Fig. 11 Measured deformation in steps of $50 \mu \mathrm{m}$.

an initial path unbalance of $9 \mathrm{~mm}$. Deformation is applied with the help of a micrometer screw attached to one of the anchorage points of the measurement fiber. Measured results are found to match with theoretical expectations to within $99.8 \%$, and the standard deviation of error is 0.007 $\mathrm{mm}$.

\section{Experiments and Discussion}

Experimental implementation of this technique was carried out using an Anritsu SLED at $1300 \mathrm{~nm}$ with a typical output power of $1 \mathrm{~mW}$ and an external integrated-optics MachZehnder-based amplitude modulator, with a modulation bandwidth of $3 \mathrm{GHz}$. Since modulation frequency and path unbalance in the sensor are closely interrelated and the latter is of the order of $1 \mathrm{~mm}$ in standard sensors, the subcarrier frequency should be in the rf range. Given the difficulties involved in working at very high frequencies, we have implemented an experimental arrangement to work at moderate frequencies, which has dictated employing SOFO sensors configured with a path unbalance of $10 \mathrm{~mm}$. The translation of these developments to higher frequencies will significantly help in improving the sensitivities that can be obtained by the method.

In order to introduce a longitudinal deformation, we used a shaker with maximum vibration amplitude of few centimeters and a bandwidth of few hundred hertz. Since the shaker vibrates only in its longitudinal direction, it was attached to one of the anchorage pieces with the help of a pulley as shown in Fig. 12. This arrangement permits introduction of a periodic deformation of controlled frequency but of unknown amplitude.

A commercial Polytec Laser Doppler vibrometer ${ }^{16}$ was used for comparison with the measured results. The vibrometer, a punctual noncontact sensor that measures the velocity of a moving surface, consists of an optical sensor head and a controller, with a maximum sensitivity of 5 $(\mathrm{mm} / \mathrm{s}) / \mathrm{V}$. We employed it for measuring the velocity of the moving anchorage point. The velocity signal was then numerically integrated to obtain the displacement of the piece.

Tests were performed at several frequencies. Figure 13 shows the measured deformation of a sinusoidal excitation at $4 \mathrm{~Hz}$. The peak-to-peak deformation amplitude in this 


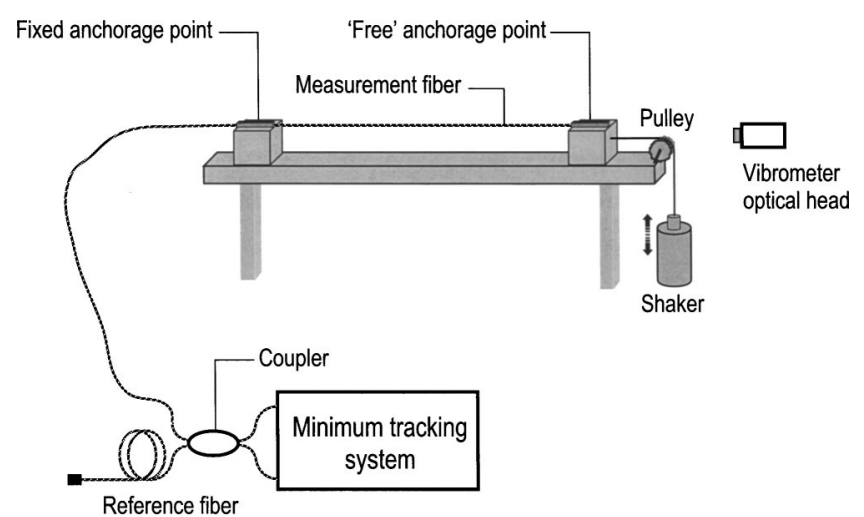

Fig. 12 Experimental arrangement for dynamic measurements with a sensor of $1.8-\mathrm{m}$ active length.

experiment was of the order of $300 \mu \mathrm{m}$. Results show that in the case of large deformations the agreement between the two measurement systems is very good.

Tests have been performed to study the frequency behavior of the minimum tracking system. Figure 14 shows the response of the system to deformations at $30 \mathrm{~Hz}$. Although the squared-off valleys and peaks indicate that the sampling frequency was not sufficiently high, the results obtained are encouraging. The peak-to-peak deformation amplitude is of the order of $70 \mu \mathrm{m}$. All measurements performed by us were obtained by applying a periodic deformation to the measurement fiber. We expect the system to give reliable results in the measurement of random deformations as well.

Experiments performed by us were limited to dynamic measurements up to $50 \mathrm{~Hz}$. Results show that the proposed system offers satisfaction as far as sensitivity, amplitude, and frequency of dynamic measurements in civil engineering structures are concerned.

As already shown in previous sections, the effect of strain-induced losses and divergence of reflectivity values in the sensor response is to make the minimum flatter. Although, in principle, this phenomenon should not influence the detection of its frequency position, it has been observed that in practice this was not completely true. Tests per-

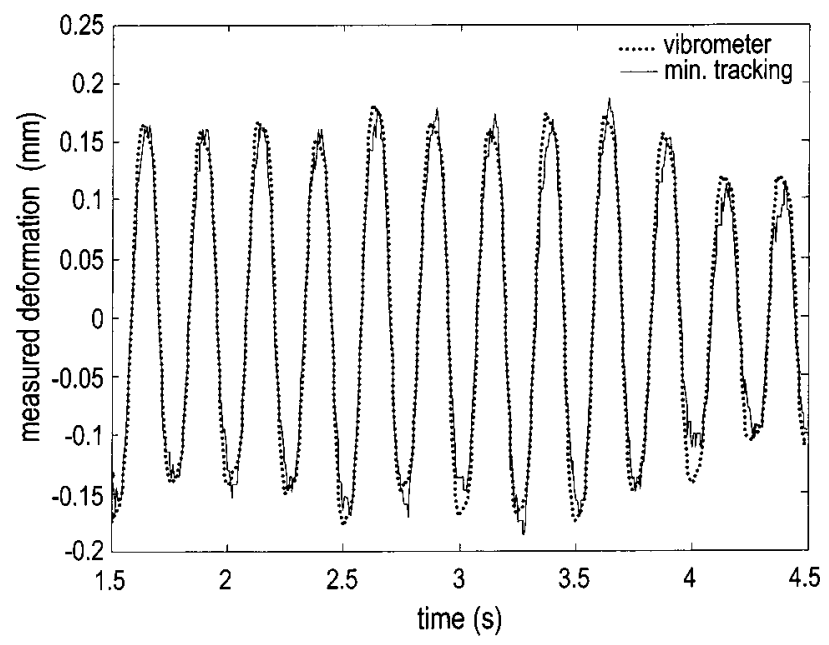

Fig. 13 Measured deformation for an excitation of $4 \mathrm{~Hz}$.

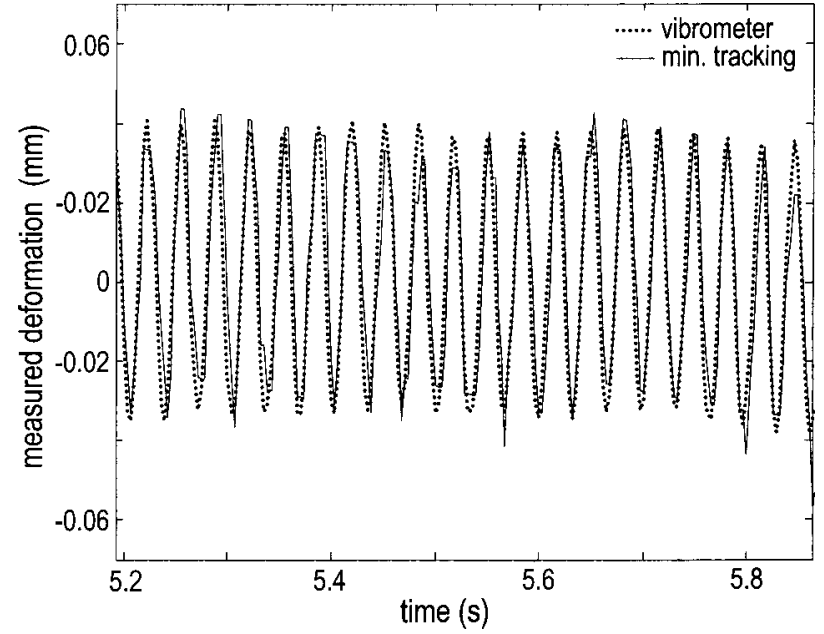

Fig. 14 Response to an excitation of $30 \mathrm{~Hz}$.

formed for different $k \alpha_{m}^{2}$ have shown that measurements obtained below a certain value of the modulation depth could not be considered reliable. The minimum tends to become too broad and thus cannot be characterized by a unique frequency. We have also observed through experiments that the tracking system becomes ineffective for a ratio between reflected intensities lower than 0.55 .

Let us define the measurement sensitivity of the method, in terms of the noise-equivalent deformation (NED), as the weakest deformation that produces a signal-to-noise ratio (SNR) of 1 at $1 \mathrm{~Hz}$. With the current bandwidth, SNR $=1$ is obtained for $10-\mu \mathrm{m}$ deformation. Since the bandwidth of the system is limited to $330 \mathrm{~Hz}$ by the lock-in amplifier,

$\mathrm{NED}=0.55 \mu \mathrm{m} \mathrm{Hz}{ }^{-1 / 2}$.

This sensitivity can be improved by further processing (filtering and amplifying) of the error signal, which fortunately does not have any influence on the feedback loop.

\section{Conclusions}

We have presented a fiber sensing Michelsoninterferometer setup based on amplitude modulation of a broadband source for the measurement of dynamic deformations. The new technique shows sensitivity better than $10 \mu \mathrm{m}$ with an adequate postfiltering stage in the error signal, a measurement range of $11 \mathrm{~mm}$ with $5 \mathrm{~mm}$ in compression and $6 \mathrm{~mm}$ in elongation of the measurement fiber, and a frequency response up to $100 \mathrm{~Hz}$. We expect the system to be useful for measuring the dynamic response of structures under dynamic loads, for instance, traffic, wind, and earthquakes. In this context, the results should be analyzed to obtain data about the load patterns and fatigue and for system identification through modal analysis.

The optical fiber sensing device proposed by us is robust, and its current performance looks more than sufficient for monitoring most dynamic phenomena encountered in large-scale civil engineering structures. 


\section{Acknowledgment}

The research described in this paper is funded by the Commission for Technology and Innovation of the Swiss government.

\section{References}

1. E. Udd, Ed., Fiber Optic Smart Structures, Wiley, New York (1995).

2. D. Inaudi, A. Elamari, L. Pflug, N. Gisin, J. Breguet, and S. Vurpillot, "Low-coherence interferometry deformation sensors for the monitoring of civil engineering structures," Sens. Actuators A 44, 125-130 (1994).

3. M. Takeda and T. Horiguchi, "Advances in optical time domain reflectometry," J. Lightwave Technol. 7, 1217-1223 (1989).

4. J. P. Dakin, "Multiplexed and distributed optical fiber sensor system," in The Distributed Fiber Optic Sensing Handbook, J. P. Dakin, Ed., IFS Publications, UK (1990).

5. S. Vurpillot, N. Casanova, D. Inaudi, and P. Kronenberg, "Bridge spatial displacement monitoring with 100 fiber optic deformation sensors: sensor network and preliminary results," Proc. SPIE 3043, 51-56 (1997).

6. B. Glisic, D. Inaudi, P. Kronenberg, S. Lloret, and S. Vurpillot, Special sensors for deformation measurements of different construction materials and structures, Proc. SPIE 3670, 505-513 (1999).

7. E. Fischer, E. Dalhoff, S. Heim, U. Hofbauer, and H. J. Tiziani, "Absolute interferometric distance measurement using a FMdemodulation technique," Appl. Opt. 34, 5589-5594 (1995).

8. A. J. den Boef, "Interferometric rangefinger using a frequency modulated laser diode," Appl. Opt. 26, 4545-4550 (1987).

9. P. Guaita, C. Mariottini, M. Martinelli, A. Melloni, F. Trave, and G. Vegetti, "Array of 32 optical fibre strain gauges deployment on a sea platform," Presented at OFS 2000, Venice (2000).

10. D. A. Jackson," "Monomode optical fibre interferometers for precision measurement," J. Phys. E 18, 981-1001 (1985).

11. H. Lamela and E. García, "Experimental evaluation of sensitivity enhancement achieved by heterodyne optical detection in AMCW laser rangefinders for machine vision," in IECON '98, Vol. 3, pp. 12901293, (1998).

12. J. Hancock, D. Langer, M. Hebert, R. Sullivan, D. Ingimarson, E. Hoffman, M. Mettenleiter, and C. Froelich, "Active laser radar for high-performance measurement," Proc. IEEE, 1465-1470 (May 1998).

13. D. Marcuse, "Curvature loss formula for optical fibers," J. Opt. Soc. Am. 66, 216-220 (1976)

14. D. Marcuse, "Bend loss of slab and fiber modes computed with diffraction theory," IEEE J. Quantum Electron. 29, 2957-2961 (1993).

15. D. Inaudi, "Fiber optic sensor network for the monitoring of civil engineering structures," $\mathrm{PhD}$ Thesis, No. 1612, EPFL, Lausanne, Switzerland (1997).

16. Polytec, www.polytec.com

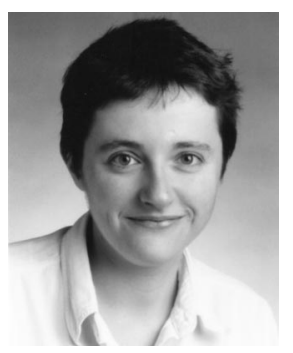

Sandra LLoret received her degree in telecommunication engineering from the Polytechnic University of Catalunya (UPC) in Barcelona in 1996. Since then she has been working at the Laboratory of Stress Analysis (IMAC) of the Swiss Federal Institute of Technology in Lausanne, where she received her $\mathrm{PhD}$ degree in 2001 . She is working on the application of fiber optic sensors in the field of civil engineering smart structures, and her area of interest is dynamic deformation measurements.

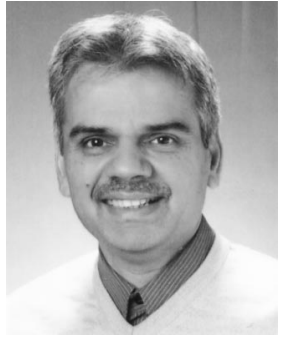

Pramod Rastogi joined the Swiss Federal Institute of Technology, Lausanne, in 1978. His research activities are principally in holographic interferometry, speckle metrology, phase shifting, and moiré. He has published five books in optical metrology, entitled Holographic InterferometryPrinciples and Methods (Springer-Verlag, Heidelberg, 1994); Optical Measurement Techniques and Applications (Artech Book House, London, 1998); Photomechanics (Springer-Verlag, Heidelberg, 2000); Trends in Optical Nondestructive Testing and Inspection (Elsevier, Oxford, 2000); and Digital Speckle Pattern Interferometry and Related Techniques (John Wiley \& Sons, Chichester, 2001). Dr. Rastogi is a Fellow of the Optical Society of America (OSA) and a Fellow of SPIE, and the co-editor-in-chief of the international journal of Optics and Lasers in Engineering published by Elsevier, Oxford.

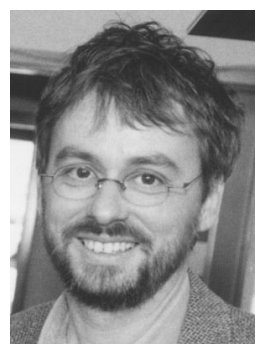

Luc Thévenaz received the MSc degree in astrophysics from the Observatory of Geneva, Switzerland, in 1982 and the PhD degree in physics from the University of Geneva, Switzerland, in 1988. He developed at that time his field of expertise, namely fiber optics. In 1988 he joined the Swiss Federal Institute of Technology of Lausanne (EPFL), where he leads a research group involved in photonics, in particular fiber optics and optical sensing. His research topics include electrical current fibre sensors, Brillouinscattering fiber sensors, fiber nonlinearity measurement techniques, and laser diode spectroscopy of gases. In 1991, he visited the PUC University in Rio de Janeiro, Brazil, and stayed at Stanford University, USA, where he participated in the development of a Brillouin laser gyroscope. In 1998 and 1999 he visited the Korea Advanced Institute of Science and Technology in Taejon, South Korea, where he worked on fiber laser current sensors.

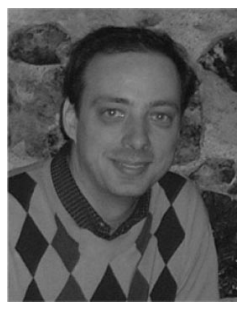

Daniele Inaudi received a degree in physics at the Swiss Federal Institute of Technology in Zurich in 1992. His graduation work was centered on the theoretical and experimental study of the polarization state of the emission of external-grating diode lasers and was awarded the ETHZ medal. $\mathrm{He}$ received his $\mathrm{PhD}$ degree from the Swiss Federal Institute of Technology in Lausanne in 1997. He was group leader of the SOFO project, a multidisciplinary research program including optics, civil engineering, and computer science, which aimed at applying fiber optic smart sensing to civil structural monitoring. Daniele Inaudi is cofounder and director of SMARTEC, a company active in fiber optic smart sensing. 